\title{
Physicochemical characteristics of Nigella seed (Nigella sativa L.) oil as affected by different extraction methods.
}

\begin{abstract}
The physicochemical properties of crude Nigella seed (Nigella sativa L.) oil which was extracted using Soxhlet, Modified Bligh-Dyer and Hexane extraction methods were determined. The effect of different extraction methods which includes different parameters, such as temperature, time and solvent on the extraction yield and the physicochemical properties were investigated. The experimental results showed that temperature, different solvents and extraction time had the most significant effect on the yield of the Nigella oil extracts. The fatty acid (FA) compositions of Nigella seed oil were further analyzed by gas chromatography to compare the extraction methods. The C16:0, C18:1 and C18:2 have been identified to be the dominant fatty acids in the Nigella seed oils. However, the main triacylglycerol (TAG) was LLL followed by OLL and PLL. The FA and TAG content showed that the composition of the Nigella seed oil extracted by different methods was mostly similar, whereas relative concentration of the identified compounds were apparently different according to the extraction methods. The melting and crystallization temperatures of the oil extracted by Soxhlet were -2.54 and $-55.76{ }^{\circ} \mathrm{C}$, respectively. The general characteristics of the Nigella seed oil obtained by different extraction methods were further compared. Where the Soxhlet extraction method was considered to be the optimum process for extracting Nigella seed oil with a higher quality with respect to the other two processes.
\end{abstract}

Keyword: Fatty acid profile; Nigella seed oil; Oil extraction methods; Physicochemical characteristics. 